Radial and Nonradial Pulsations as Probes of Stellar Physics

ASP Conference Series, Vol. 259, 2002

C. Aerts, T.R. Bedding, \& J. Christensen-Dalsgaard, eds.

\title{
Search for New $\gamma$ Doradus Variable Stars in Open Clusters: Praesepe and $\alpha$ Persei
}

\author{
S. Martín \\ Osservatorio Astronomico di Brera, Via Bianchi 46, I-23807 Merate, \\ Italy \\ E. Rodríguez \\ Instituto de Astrofísica de Andalucía, CSIC, P.O. Box 3004, E-18080 \\ Granada, Spain
}

\begin{abstract}
Photometric observations of A-F stars in the $u v b y \beta$ system have been performed in order to detect $\gamma$ Doradus variables in open clusters of different ages. In the clusters Praesepe and $\alpha$ Per, several stars show some type of variability but only three of them, KW370 (Praesepe) and HE104 and HE876 ( $\alpha$ Per), can be classified as $\gamma$ Doradus-type pulsators.
\end{abstract}

\section{Discussion}

$\alpha$ Persei $(\log ($ age $)=7.90)$ and Praesepe $(\log ($ age $)=8.84)$ are, together with other clusters as the Pleiades $(\log ($ age $)=7.92)$ (Martín \& Rodríguez, 2000), part of a study on the presence of $\gamma$ Doradus pulsating stars in open clusters with different ages.

The observations were performed using the $0.90 \mathrm{~m}$ telescope at Observatorio de Sierra Nevada, Spain, by means of a Strömgren six-channel simultaneous photometer. The stars checked out were selected from a set of A-F stars $\left(2 .^{m} 650\right.$ $<\beta<2{ }^{m} 800$ ) with $V<12 \cdot^{m} 00$. Both restrictions guarantee a good position in the H-R diagram, where the $\gamma$ Dor variables are being discovered, and a good signal-to-noise ratio. The sequence of observations per night, independently for each cluster, was the following: $C 1-C 2-C 3$-first group of stars-C1-C2-C3-second group of stars, $C 1-C 2-C 3$, etc. The size of each group depends on the number of objects in each campaign and on the conditions of the sky in each night. In the case of $\alpha$ Persei, 18 stars were measured using the uvby filters during four nights in 1996 and only the most interesting (13) were again observed in 1997 during five nights. Praesepe was observed during 1997 and 1999 with the number of objects being reduced from 16 to 9 in the second year.

Frequency analyses were carried out on the measured stars. The method used is described in Bossi \& Nuñez (2002). When the objects of $\alpha$ Persei were taken into account, significant periodicities were found only for the stars HE104 and HE876 (Heckmann et al., 1956). Both stars are monoperiodic with frequencies $f=3.12355 \mathrm{~d}^{-1}$ and $f=1.54409 \mathrm{~d}^{-1}$, respectively, and amplitudes of $0 .^{m} 009$ and $0 .^{m} 012$, from peak to peak in the $V$ filter. These periodicities are typical of 
the $\gamma$ Doradus-type pulsators. The results are consistent in the four filters, with amplitude-ratios between different bands typical of pulsating stars in this region. In the case of Praesepe, only one star (KW370, Klein-Wassink 1927) was found to be variable, but with three frequencies: $f_{1}=1.30679 \mathrm{~d}^{-1}, f_{2}=1.40450 \mathrm{~d}^{-1}$ and $f_{3}=1.78272 \mathrm{~d}^{-1}$ and much larger amplitude (full mean amplitude of about $\left.0 .^{m} 04\right)$.

Due to the small number of measurements collected during these campaigns, the results are not definitive: different and/or additional periods might be present in any of the stars. However, in spite of our limited datasets, from the variations of the colour indices and their positions in H-R diagram we can consider to HE104 and HE876 ( $\alpha$ Persei) and KW370 (Praesepe) as $\gamma$ Doradus pulsating stars. Anyway, new data are needed in order to get definite variability models.

\section{References}

Bossi, M. \& Nuñez, N.S. 2002, in preparation

Heckmann, V.O., Dieckvoss, W., \& Kox, H. 1956, Astron. Nachr., 283, 109 Klein-Wassink, W.J. 1927, Publ. Kapteyn Astron. Lab. No. 41

Martín, S. \& Rodríguez, E. 2000, A\&A, 358, 287 


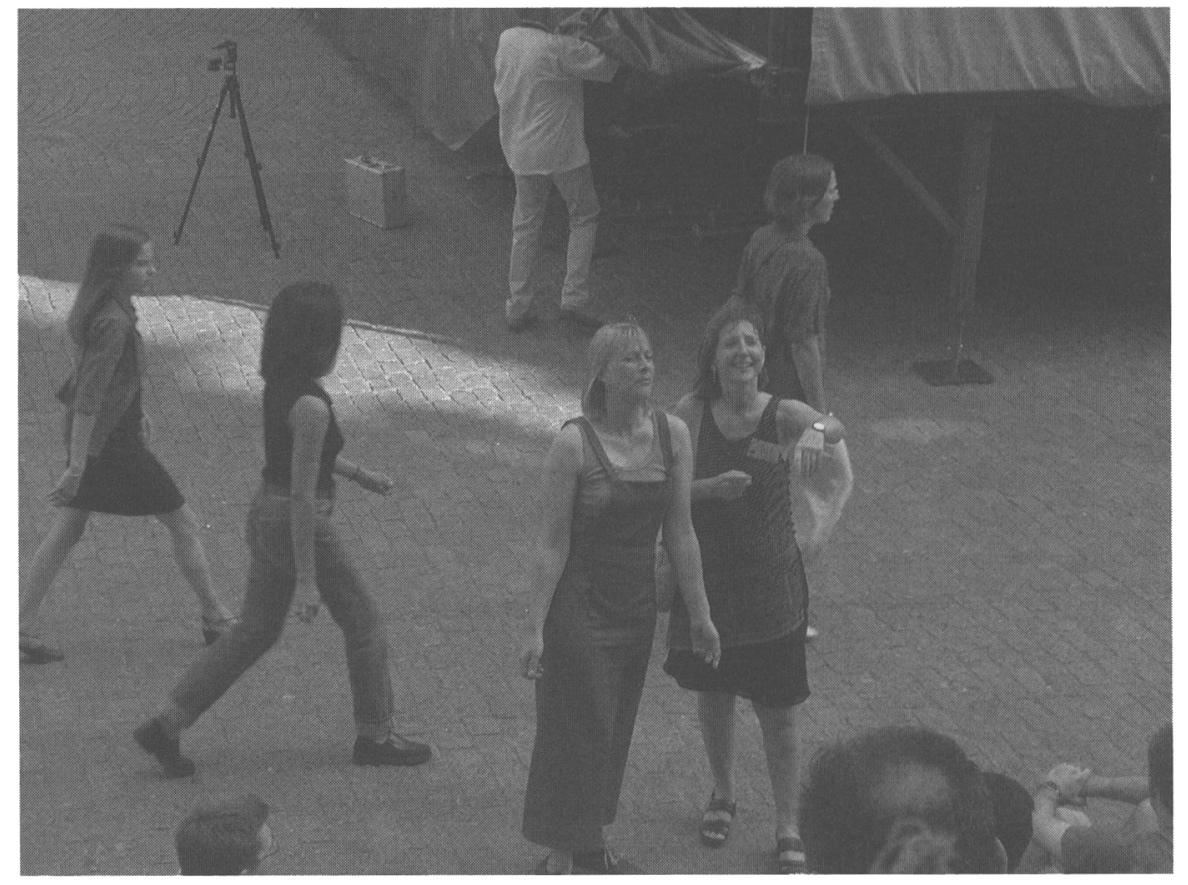

The photographer and the chairwoman of the LOC are trying very hard to get all the participants in a good position for the group picture and to keep them there for a few minutes. You can see the result of their struggle on page xxii. 